\title{
SINGULAR PERTURBATIONS AND THE TRANSITION FROM THIN PLATE TO MEMBRANE
}

\author{
ZEEV SCHUSS
}

ABSTRACT. The equation

$$
\frac{E h^{2}}{12\left(1-\sigma^{2}\right)} \Delta^{2} w-\sum_{i, j=1}^{2} \frac{\partial}{\partial x_{i}}\left(\sigma_{i j} \frac{\partial w}{\partial x_{j}}\right)=f
$$

describing the normal displacement $w$ of a thin elastic plate of thickness $h$ under uniform tension in equilibrium is considered. It is shown that if the displacement and its normal derivative on the edge of the plate are bounded uniformly with respect to $h$ then the solution $u$ of the membrane equation

$$
-\sum_{i, j=1}^{2} \frac{\partial}{\partial x_{i}}\left(\sigma_{i j} \frac{\partial u}{\partial x_{j}}\right)=f
$$

with the same boundary values as $w$ approximates the displacement throughout the plate in the $L^{2}$ sense. Herein, the rate

$$
\iint_{\Omega}|w(x)-u(x)|^{2} d x \in C h^{2} \iint_{\Omega}|f(x)|^{2} d x
$$

is given, where $C$ is a constant independent of $h$ and $f$, and $\Omega$ in the face of the plate. This extends the results of A. Friedman [6] and F. John [10] up to the boundary and improves the rate of convergence in (*) given by J. L. Lions [12] and W. M. Greenlee [7] from $h$ to $h^{2}$.

The equation describing the normal displacement $w\left(x_{1}, x_{2}\right)$ of a thin elastic plate of thickness $h$ in equilibrium is [11]

$$
\frac{E h^{2}}{12\left(1-\sigma^{2}\right)} \Delta^{2} w-\sum_{i, j=1}^{2} \frac{\partial}{\partial x_{i}}\left(\sigma_{i j} \frac{\partial w}{\partial x_{j}}\right)=f .
$$

Here as usual, $E$ denotes Young's modulus, $\sigma$ Poisson's ratio, $\sigma_{i j}$ are the components of the stress in the plate and $f$ is the external force per unit area. Since the effect of the forces or constraints acting on the edge of the plate can be achieved by replacing $w$ by $w-\Phi$ we may assume that the plate does not undergo any deformation at the edge; thus the displacement is caused solely by forces acting on the faces of the plate. The corresponding displacement of a membrane is governed by the second order equation [11]

$$
-\sum_{i, j=1}^{2} \frac{\partial}{\partial x_{i}}\left(\sigma_{i j} \frac{\partial u}{\partial x_{j}}\right)=f .
$$

As mentioned in [10], replacing (I) by (II) can in general only be justified

Received by the editors December 3, 1974.

AMS (MOS) subject classifications (1970). Primary 35B25, 73K10; Secondary 35J40, 73K 15.

Key words and phrases. Singular perturbations, higher order elliptic boundary value problems, thin plates and membranes.

(1) American Mathematical Society 1976 
when the plate is under tension, i.e. when the $\sigma_{i j}$ form the coefficients of a positive definite quadratic form. The special case $f=0$ and $w$ and its derivative is given on the edge of the plate was considered by F. John [10]. For general constant tension $\sigma_{i j}$ he obtains the interior estimate

$$
w(\mathbf{y})-u(\mathbf{y})=O\left(\frac{h^{2}}{r^{2}} W\right) \quad\left(W=\sup _{\mathbf{x} \in \Omega}|w(\mathbf{x})|\right)
$$

where $u(y)$ is a solution of (II) determined by the boundary values of $w$ and its derivatives up to order 3 on the edge of the plate. Here $r$ denotes the distance from $\mathbf{y}$ to the edge. Thus $u$ is also a function of $h$. In this paper we show that if the displacement and its normal derivatives on the edge of the plate are bounded uniformly with respect to $h$ then the membrane solution with the same boundary values as $w$ approximates the displacement throughout the plate in the $L^{2}$ sense. Herein, the rate

$$
\iint_{\Omega}|w(\mathbf{x})-u(\mathbf{x})|^{2} d \mathbf{x} \leqslant C h^{2} \iint_{\Omega}|f(\mathbf{x})|^{2} d \mathbf{x}
$$

is given, where $C$ is a constant independent of $h$ and $f$, and $\Omega$ is the face of the plate.

Similar estimates were derived by J. L. Lions [12] where the $H^{1}(\Omega)$ norm of $w-u$ is shown to be $O\left(h^{1 / 2}\|f\|_{L^{2}(\Omega)}\right)$. A. Friedman [6] derived (0.2) in the interior of $\Omega ; \mathrm{W}$. Greenlee [7] obtained results similar to [12], C. Bardos and D. and $\mathrm{H}$. Brezis [2] prove the strong convergence in $L^{2}(\Omega)$ of $w$ to $u$; they do not obtain, however, estimates similar to $(0.2)$. M. I. Vishik and L. A. Liusternik [14] expand $w$ asymptotically in $h$ under some differentiability assumptions on $f$, but they do not estimate the rate of convergence of $w$ to $u$.

The author wishes to express his gratitude to the referee for his valuable comments.

1. Preliminary results and estimates in a half plane. Let $\Omega$ be a bounded domain in $R^{2}$ with boundary $\partial \Omega$ in the class $C^{\infty}$. We denote by $n$ the outward normal to $\partial \Omega$. We may assume that $\sigma_{11}=1$ and use $\varepsilon=$ $E h^{2} / 12\left(1-\sigma^{2}\right)$. Set

$$
\begin{aligned}
& L=\varepsilon \Delta^{2}-\sum_{i, j=1}^{2} \sigma_{i j} \frac{\partial^{2}}{\partial x_{i} \partial x_{j}}, \\
& M=-\sum_{i, j=1}^{2} \sigma_{i j} \frac{\partial^{2}}{\partial x_{i} \partial x_{j}} .
\end{aligned}
$$

Let $w(\mathbf{x})=w\left(x_{1}, x_{2}\right)$ be the solution of

$$
\begin{aligned}
L w & =f \quad \text { in } \Omega, \\
w & =\partial w / \partial n=0 \quad \text { on } \partial \Omega
\end{aligned}
$$

and let $u(\mathbf{x})$ be the solution of

$$
\begin{array}{rlrl}
M u=f & \text { in } \Omega, \\
u=0 & & \text { on } \partial \Omega .
\end{array}
$$

Let $\phi(\mathbf{x})$ be a smooth function in $\Omega$ which is equal to the distance from the boundary near $\partial \Omega$ and $\phi=1$ away from $\partial \Omega$. We use $H^{k}(\Omega)$ to denote the 
Sobolev space [5] of all square integrable functions in $\Omega$ that have square integrable partial derivatives up to order $k$ in $\Omega$, and for $v \in H^{k}(\Omega)$ the norm is given by

$$
\|v\|_{H^{k}(\Omega)}^{2}=\sum_{|\alpha|<k} \iint_{\Omega}\left|D^{\alpha} v\right|^{2} d \mathbf{x}
$$

LEMMA 1.1. There exists a positive constant $C$ such that

$$
\begin{gathered}
\varepsilon\|w\|_{H^{2}(\Omega)}^{2}+\|w\|_{H^{1}(\Omega)}^{2} \leqslant C\|f\|_{L^{2}(\Omega)}^{2}, \\
\varepsilon^{2}\|\phi w\|_{H^{4}(\Omega)}^{2}+\|\phi u\|_{H^{2}(\Omega)}^{2} \leqslant C\|f\|_{L^{2}(\Omega)}^{2}
\end{gathered}
$$

for all $\varepsilon>0$.

PRoof. Inequality (1.4) is easily obtained using integration by parts (cf. D. Huet [9] or A. Friedman [6]). To obtain (1.5) we multiply equation (1.1) by $\phi^{2} M w$ and integrate by parts. Using (1.4) we obtain

$$
\|\phi M w\|_{L^{2}(\Omega)} \leqslant C\|f\|_{L^{2}(\Omega)}
$$

It follows from [13], [1], or [3] and (1.4) that

$$
\|\phi w\|_{H^{2}(\Omega)} \leqslant C\|f\|_{L^{2}(\Omega)} .
$$

Now we multiply (1.1) by $\phi$ and obtain

$$
\varepsilon\left\|\phi \Delta^{2} w\right\|_{L^{2}(\Omega)} \leqslant C\left(\|\phi M w\|_{L^{2}(\Omega)}+\|f\|_{L^{2}(\Omega)}\right) .
$$

Using [13], [1], [3] again we get (1.5).

COROLlaRY. Under the assumptions of Lemma 1.1

$$
\varepsilon\|w\|_{H^{3}(\Omega)} \leqslant C\|f\|_{L^{2}(\Omega)}
$$

and

$$
\varepsilon^{1 / 2}\|\phi w\|_{H^{3}(\Omega)} \leqslant C\|f\|_{L^{2}(\Omega)} .
$$

Proof. Using (1.4), (1.1) and the uniform ellipticity of $\Delta^{2}$ we see [5] that

$$
\|w\|_{H^{2}(\Omega)} \leqslant C \varepsilon^{-1 / 2}\|f\|_{L^{2}(\Omega)} \text { and }\|w\|_{H^{4}(\Omega)} \leqslant C \varepsilon^{-3 / 2}\|f\|_{L^{2}(\Omega)} \text {. }
$$

Hence using interpolation [12] we get (1.6). Similar arguments lead to (1.7).

Let $R_{+}^{2}$ be the half plane $\left\{\left(x_{1}, x_{2}\right) \in R^{2} \mid x_{1} \in R^{1}, x_{2}>0\right\}$.

Lemma 1.2. Assume $f(x) \in L^{2}\left(R_{+}^{2}\right)$ and $f\left(x_{1}, x_{2}\right)=0$ for $x_{2}>K$. Let $w$ be the solution of (1.1) in $R_{+}^{2}$ satisfying the Dirichlet boundary conditions

$$
w\left(x_{1}, 0\right)=\partial w\left(x_{1}, 0\right) / \partial x_{2}=0, \quad w \text { is bounded in } R_{+}^{2},
$$

and let $u(x)$ be the solution of (1.2) in $R_{+}^{2}$ satisfying

$$
u\left(x_{1}, 0\right)=0, \quad u \text { is bounded in } R_{+}^{2} .
$$

Then there exist positive constants $\varepsilon_{0}$ and $C$ such that

$$
\|w-u\|_{L^{2}\left(R_{+}^{2}\right)} \leqslant C \varepsilon^{1 / 2}\|f\|_{L^{2}\left(R_{+}^{2}\right)}
$$

for all $0<\varepsilon \leqslant \varepsilon_{0}$, C is independent of $\varepsilon$ and $f$ but depends on $K$. 
Proof. Assume $f \in C_{0}^{\infty}\left(R_{+}^{2}\right)$. Using the Fourier transform in $x_{1}$ we obtain for $w$ and $h$ the ordinary differential equations

$$
\begin{gathered}
{\left[\varepsilon\left(-\xi_{1}^{2}+\frac{\partial^{2}}{\partial x_{2}^{2}}\right)^{2}-\left(-\xi_{1}^{2}+2 \sigma_{12} i \xi_{1} \frac{\partial}{\partial x_{2}}+\sigma_{22} \frac{\partial^{2}}{\partial x_{2}^{2}}\right)\right] \hat{u}\left(\xi_{1}, x_{2}\right)} \\
=\hat{f}\left(\xi_{1}, x_{2}\right)
\end{gathered}
$$

and

$$
\left[-\xi_{1}^{2}+2 \sigma_{12} i \xi_{1} \frac{\partial}{\partial x_{2}}+\sigma_{22} \frac{\partial^{2}}{\partial x_{2}}\right] \hat{u}\left(\xi_{1}, x_{2}\right)=\hat{f}\left(\xi_{1}, x_{2}\right) .
$$

The characteristic equation for (1.11) is

$$
\varepsilon\left(\rho^{2}-\xi_{1}^{2}\right)^{2}-\left(\sigma_{22} \rho^{2}+2 \sigma_{12} i \xi_{1} \rho-\xi_{1}^{2}\right)=0 .
$$

Setting $\rho=\xi_{1} r$ we obtain

$$
\delta\left(r^{2}-1\right)^{2}-\left(\sigma_{22} r^{2}+2 \sigma_{12} i r-1\right)=0
$$

where $\delta=\varepsilon \xi_{1}^{2}$.

We consider the three cases: (i) $\delta<\delta_{1}$, (ii) $\delta>\delta_{2}$ and (iii) $\delta_{1} \leqslant \delta \leqslant \delta_{2}$, where $\delta_{1}$ is a sufficiently small positive number and $\delta_{2}$ is a sufficiently large number independent of $\varepsilon$ and $\xi_{1}$. In case (i) we set $r=s^{-1}$ so that (1.13) becomes

$$
\delta\left(1-s^{2}\right)^{2}-\left(\sigma_{22} s^{2}+2 \sigma_{12} i s^{3}-s^{4}\right)=0 .
$$

Since the equation $\sigma_{22} s^{2}+2 b i s^{3}-s^{4}=0$ has the double root $s=0$ and two simple roots $s_{1}$ and $s_{2}$, we have [8] the expansions

$$
s= \pm \delta^{1 / 2}[1+o(1)] \text { as } \delta \rightarrow 0
$$

and

$$
s=s_{j}[1+O(\delta)] \text { as } \delta \rightarrow 0, j=1,2 .
$$

Therefore there exists a positive constant $\delta_{1}$ such that the roots $\rho_{j}$ of (1.11) have the representation

$$
\begin{aligned}
& \rho_{1}=\xi_{1}\left(i \sigma_{12}-\left(\sigma_{22}-\sigma_{12}^{2}\right)^{1 / 2}+\delta \alpha\right) \equiv \xi_{1}(i b-D+\alpha \delta) \equiv i b_{\varepsilon} \xi_{1}-D_{\varepsilon} \xi_{1}, \\
& \rho_{2}=\xi_{1}(i b+D+\delta \alpha) \equiv i b_{\varepsilon} \xi_{1}+D_{\varepsilon} \xi_{1}, \\
& \rho_{3}=\varepsilon^{-1 / 2}(1+\delta \beta) \operatorname{sgn} \xi_{1} \equiv i b_{\varepsilon}^{\prime} \xi_{1}-D_{\varepsilon}^{\prime} \xi_{1}, \\
& \rho_{4}=-\varepsilon^{-1 / 2}(1+\delta \beta) \operatorname{sgn} \xi_{1} \equiv i b_{\varepsilon}^{\prime} \xi_{1}+D_{\varepsilon}^{\prime} \xi_{1},
\end{aligned}
$$

where $\alpha$ and $\beta$ are bounded functions of $\varepsilon$ and $\xi_{1}$. In case (ii) we get similarly

$$
\rho_{j}= \pm \varepsilon^{-1 / 2}\left(1 \pm \delta^{-1 / 2} \gamma\right), \quad j=1,2,3,4
$$

where $\gamma$ is a bounded function of $\varepsilon$ and $\xi_{1}$.

Finally in case (iii)

$$
\rho_{j}=\xi_{1} r_{j}, \quad j=1,2,3,4,
$$

where $r_{j}$ are bounded functions of $\varepsilon$ and $\xi_{1}$. We have $\rho_{1}=-\bar{\rho}_{2}$ and $\rho_{3}=-\bar{\rho}_{4}$. The solution $u$ of (1.2), (1.9) is given by 


$$
\hat{u}\left(\xi_{1}, x_{2}\right)=\int_{0}^{\infty} G\left(\xi_{1}, x_{2}, t\right) \hat{f}\left(\xi_{1}, t\right) d t
$$

where

$$
G\left(\xi_{1}, x_{2}, t\right)=-\left[\exp \left(i b\left|\xi_{1}\right|\left|x_{2}-t\right|-D\left|\xi_{1}\right| x_{2}\right)\right]\left(\sinh D\left|\xi_{1}\right| t / D\left|\xi_{1}\right|\right)
$$

if $x_{2} \geqslant t$, and for $x_{2}<t, G$ is defined by the relation

$$
G\left(\xi_{1}, x_{2}, t\right)=\overline{G\left(\xi_{1}, t, x_{2}\right)} .
$$

The solution $w$ of $(1.1),(1.8)$ is given by

$$
\hat{w}\left(\xi_{1}, x_{2}\right)=\int_{0}^{\infty} G_{\varepsilon}\left(\xi_{1}, x_{2}, t\right) \hat{f}\left(\xi_{1}, t\right) d t+\int_{0}^{\infty} H_{\varepsilon}\left(\xi_{1}, x_{2}, t\right) \hat{f}\left(\xi_{1}, t\right) d t
$$

where the functions $G_{\varepsilon}$ and $H_{\varepsilon}$ are defined by

$$
\begin{aligned}
G_{\varepsilon}\left(\xi_{1}, x_{2}, t\right)=- & {\left[\exp \left(i b_{\varepsilon}\left|\xi_{1}\right|\left|x_{2}-t\right|-D_{\varepsilon}\left|\xi_{1}\right| x_{2}\right)\right] /\left|\rho_{3}-\rho_{1}\right|^{2} \varepsilon } \\
& \cdot\left[\frac{\left(\rho_{4}-i b_{\varepsilon}\left|\xi_{1}\right|\right)}{\left(\rho_{4}-\rho_{1}\right)} \frac{\sinh D_{\varepsilon}\left|\xi_{1}\right| t}{D_{\varepsilon}\left|\xi_{1}\right|}-\frac{\cosh D_{\varepsilon}\left|\xi_{1}\right| t}{\rho_{4}-\rho_{1}}\right] \text { if } x_{2} \geqslant t,
\end{aligned}
$$

$$
\begin{aligned}
H_{\varepsilon}\left(\xi_{1}, x_{2}, t\right)= & G_{\varepsilon}^{\prime}\left(\xi_{1}, x_{2}, t\right)+\frac{\exp \left(-\left|\xi_{1}\right|\left(D_{\varepsilon}+D_{\varepsilon}^{\prime}\right) t\right)}{\left|\rho_{1}-\rho_{3}\right|^{2}\left|\rho_{1}-\rho_{4}\right|^{2}} \\
& \cdot\left[\left(\rho_{4}-\rho_{1}\right) \exp \left(i\left|\xi_{1}\right|\left(b_{\varepsilon}^{\prime} x_{2}-b_{\varepsilon} t\right)\right)\right. \\
& \left.-\left(\bar{\rho}_{4}-\bar{\rho}_{1}\right) \exp \left(-i\left|\xi_{1}\right|\left(b_{\varepsilon}^{\prime} t-b_{\varepsilon} x_{2}\right)\right)\right] \text { if } x_{2} \geqslant t,
\end{aligned}
$$

and for $x_{2}<t$ they are defined by the relations

$$
G_{\varepsilon}\left(\xi_{1}, x_{2}, t\right)=\overline{G_{\varepsilon}\left(\xi_{1}, t, x_{2}\right)} \text { and } H_{\varepsilon}\left(\xi_{1}, x_{2}, t\right)=\overline{H_{\varepsilon}\left(\xi_{1}, t, x_{2}\right)} \text {. }
$$

The function $G_{\varepsilon}^{\prime}$ is given by (1.15) with the roles of $\rho_{1}$ and $\rho_{3}$ exchanged. We consider case (i) first. A typical summand of $\hat{w}-\hat{u}$ is

$$
\begin{aligned}
l_{1} & =\int_{0}^{x_{2}}\left[\frac{\exp \left(i b_{\varepsilon}\left|\xi_{1}\right|\left|x_{2}-t\right|-D_{\varepsilon}\left|\xi_{1}\right| x_{2}\right)}{\left(\rho_{4}-\rho_{1}\right)\left|\rho_{3}-\rho_{1}\right|^{2} \varepsilon} \cosh D_{\varepsilon}\left|\xi_{1}\right| t\right] \hat{f}\left(\xi_{1}, t\right) d t \\
& \equiv \int_{0}^{x_{2}} W\left(\xi_{1}, x_{2}, t\right) \hat{f}\left(\xi_{1}, t\right) d t
\end{aligned}
$$

The denominator is equal to $\varepsilon^{-1 / 2}[1+O(\delta)]$ if $\delta$ is small, $\delta \leqslant \delta_{1}$ say, therefore

$$
\left|l_{1}\right| \leqslant C \varepsilon^{1 / 2} \int_{0}^{x_{2}}\left[\exp \left(-D_{\varepsilon}\left|\xi_{1}\right| x_{2}\right)\right]\left[\cosh \left(D_{\varepsilon}\left|\xi_{1}\right| t\right)\right]\left|\hat{f}\left(\xi_{1}, t\right)\right| d t
$$

and similarly,

$$
\begin{aligned}
& \left|l_{2}\right|=\left|\int_{x_{2}}^{\infty} \overline{W\left(\xi_{1}, t, x_{2}\right)} \hat{f}\left(\xi_{1}, t\right) d t\right| \\
& \leqslant C \varepsilon^{1 / 2} \int_{x_{2}}^{\infty}\left[\exp \left(-D_{\varepsilon}\left|\xi_{1}\right| t\right)\right]\left[\cosh D_{\varepsilon}\left|\xi_{1}\right| x_{2}\right]\left|\hat{f}\left(\xi_{1}, t\right)\right| d t .
\end{aligned}
$$

Thus 


$$
\left|l_{1}+l_{2}\right| \leqslant C \varepsilon^{1 / 2} \int_{0}^{K} K\left(x_{2}, t\right)\left|\hat{f}\left(\xi_{1}, t\right)\right| d t
$$

since supp $f\left(x_{1}, x_{2}\right) \subset\left\{0 \leqslant x_{2} \leqslant K\right\}$. To estimate $\left\|l_{1}+l_{2}\right\|_{L^{2}\left(R_{+}^{2}\right)}$ we rely on the following principle: if $\int|K(x, y)| d x \leqslant C_{1}$ and $\int|K(x, y)| d y \leqslant C_{2}$ then

$$
\int\left|\int K(x, y) f(y) d y\right|^{2} d x \leqslant C_{1} C_{2} \int|f(x)|^{2} d x,
$$

which follows easily from the Riesz-Thorin convexity theorem [4]. Applying Plancherel's formula and the above principle we get

$$
\int_{-\infty}^{\infty}\left(\int_{0}^{\infty}\left|\left(l_{1}+l_{2}\right)\left(\xi_{1}, x_{2}\right)\right| d x_{2}\right)^{2} d \xi_{1} \leqslant C_{1} C_{2} \int_{-\infty}^{\infty} \int_{0}^{\infty}\left|f\left(x_{1}, x_{2}\right)\right|^{2} d x_{2} d x_{1}
$$

where

$$
C_{1}=\sup _{\xi_{1}, x_{2}} \int_{0}^{K}\left|K\left(x_{2}, t\right)\right| d t
$$

and $C_{2}$ is defined similarly. Direct computations show that

$$
C_{1}, C_{2} \leqslant C \varepsilon^{1 / 2} \sup _{\xi_{1}} \frac{1-e^{-K\left|\xi_{1}\right|}}{\left|\xi_{1}\right|}=C \varepsilon^{1 / 2} .
$$

The other terms of $\hat{w}-\hat{u}$ are similarly estimated by $C \varepsilon^{1 / 2}$. In cases (ii) and (iii) all terms are bounded by $C \varepsilon^{1 / 2}$ in $L^{2}\left(R_{+}^{2}\right)$ and $C$ is independent of $K$. In cases (iii) we use the fact $\left|\xi_{1}\right| \geqslant \delta_{1} \varepsilon^{-1 / 2}$ and $\left|r_{j}-r_{k}\right| \geqslant c>0$ if $j \neq k$. This concludes the proof of Lemma 1.2.

\section{Estimates near the boundary and main results.}

LEMMA 2. Let $w$ and $u$ be the solutions of (1.1) and (1.3) in $\Omega$ satisfying the homogeneous Dirichlet boundary conditions. Then there exists a neighborhood $U$ of $\partial \Omega$ in $\bar{\Omega}$ such that

$$
\|w-u\|_{L^{2}(U)} \leqslant C \varepsilon^{1 / 2}\|f\|_{L^{2}(\Omega)}+C\|w-u\|_{L^{2}(\Omega-U)},
$$

where $C$ and $U$ are independent of $\varepsilon$ and $f$.

Proof. For any point $\mathbf{x}_{0} \in \partial \Omega$ we can find a neighborhood $U_{0}$ of $\mathbf{x}_{0}$ in $\bar{\Omega}$ and a smooth mapping of $U_{0}$ into $B_{r}=\left\{\mathbf{x} \in R^{2}|| x_{1} \mid<r, 0 \leqslant x_{2}<r^{2}\right\}$ so that $\bar{U}_{0} \cap \partial \Omega$ is mapped onto $B_{r} \cap\left\{x_{2}=0\right\}$ and such that $\mathbf{x}_{0}$ is mapped into the origin. Then using an affine transformation of the coordinates we can write $L$ and $M$ in $B_{r}$ as follows.

$$
\begin{aligned}
\tilde{L}= & \varepsilon \Delta^{2}-\sum_{i, j=1}^{2} a_{i j} \frac{\partial^{2}}{\partial x_{i} \partial x_{j}}+\varepsilon\left[x_{1} N(\mathbf{x}, D)+x_{2} P(\mathbf{x}, D)+Q(\mathbf{x}, D)\right] \\
& +x_{1} R(\mathbf{x}, D)+x_{2} S(\mathbf{x}, D)+T(\mathbf{x}, D), \\
\tilde{M}= & -\sum_{i, j=1}^{2} a_{i j} \frac{\partial^{2}}{\partial x_{i} \partial x_{j}}+x_{1} R(\mathbf{x}, D)+x_{2} S(\mathbf{x}, D)+T(\mathbf{x}, D)
\end{aligned}
$$

where the quadratic form corresponding to $a_{i j}$ is positive definite, $a_{i j}$ is constant and $N, P, Q, R, S, T$ are partial differential operators with coefficients in $C_{0}^{\infty}\left(B_{r}\right)$ of orders $4,4,3,2,2$ and 1 respectively. Let $\psi$ be a 
function in $C_{0}^{\infty}\left(B_{r}\right)$ such that $\psi=1$ in $B_{r / 2}$. Then the functions $\psi w$ and $\psi u$ satisfy equations of the form

$$
\begin{aligned}
L \psi w & =f_{1, \varepsilon}, \\
M \psi u & =f_{1}
\end{aligned}
$$

where $L$ and $M$ are defined by $(1.1)$ and $\left(1.1^{\prime}\right)$ respectively $\left(\sigma_{i j}\right.$ is replaced by $\left.a_{i j}\right)$ and $f_{1, \varepsilon}$ and $f_{1}$ are defined by (2.2)-(2.5). We have to estimate terms of the form $\varepsilon x_{1} N w, \varepsilon x_{2} P w$, etc. We may assume that $N w=\Sigma_{|\alpha| \leqslant 4} D^{\alpha}\left(a^{\alpha}(\mathbf{x}) w\right)$ so that

$$
\begin{array}{r}
\int_{0}^{\infty} G_{\varepsilon}\left(\xi_{1}, x_{2}, t\right)\left(x_{1} N w\right)^{\wedge}\left(\xi_{1}, t\right) d t=-i \int_{0}^{\infty} G_{\varepsilon}\left(\xi_{1}, x_{2}, t\right) \frac{\partial}{\partial \xi_{1}}(N w)^{\wedge}\left(\xi_{1}, t\right) d t \\
=i \int_{0}^{\infty} \frac{\partial}{\partial \xi_{1}} G_{\varepsilon}\left(\xi_{1}, x_{2}, t\right) \sum_{|\alpha|<4}\left(i \xi_{1} \frac{\partial}{\partial x_{2}}\right)^{\alpha}\left(a^{\alpha} w\right)^{\wedge}\left(\xi_{1}, t\right) d t
\end{array}
$$

Since $\xi_{1}^{2} \partial\left(G_{\varepsilon}+H_{\varepsilon}\right) / \partial \xi_{1}$ is a bounded operator and Lemma 1.1 shows that $\varepsilon^{1 / 2}\|w\|_{H^{2}(\Omega)} \leqslant C\|f\|_{L^{2}(\Omega)}$, we see that all summands of $\varepsilon x_{1} N w$ containing two or more derivatives with respect to $x_{1}$ contribute to $L^{-1}\left(\varepsilon x_{1} N w\right)$ a quantity that is bounded by $C \varepsilon^{1 / 2}\|f\|_{L^{2}(\Omega)}$. The functions $G_{\varepsilon}+H_{\varepsilon}, \partial\left(G_{\varepsilon}+H_{\varepsilon}\right) / \partial t$ and their $\xi_{1}$ derivative vanish for $x_{2}=0$, therefore we can integrate by parts expressions containing three or four derivatives of $w$ with respect to $t$. Since $\partial^{3}\left(G_{\varepsilon}+H_{\varepsilon}\right) / \partial \xi_{1} \partial t^{2}$ is a bounded operator in $L^{2}\left(B_{r}\right)$ and $\psi w \in C_{0}^{\infty}\left(B_{r}\right)$, we see that the contribution of such derivatives to $L^{-1}\left(\varepsilon x_{1} N w\right)$ is bounded by $C \varepsilon^{1 / 2}\|f\|_{L^{2}(\Omega)}$. To estimate $\varepsilon x_{2} P w$ we use similar arguments and (1.7), and for $\varepsilon Q w$ we use integration by parts and (1.6). Writing $\psi w=L^{-1} f_{1, \varepsilon}$ and $\psi u$ $=M^{-1} f_{1}$ we see that we have to estimate expressions of the form

$$
\left(L^{-1}-M^{-1}\right) x_{1} R(\mathbf{x}, D) w \text { and } M^{-1} x_{1} R(\mathbf{x}, D)(w-u),
$$

etc. The first expression can be estimated by $C \varepsilon^{1 / 2}\|f\|_{L^{2}(\Omega)}$ using Lemma 1.2 and (1.5). To estimate the second expression we note that the function $\psi$ can be chosen so that it is independent of $x_{2}$ in $0 \leqslant x_{2} \leqslant r^{2} / 4$, and $\left|\partial \psi / \partial x_{1}\right|$ $\leqslant C / r,\left|\partial^{2} \psi / \partial x_{1}^{2}\right| \leqslant C / r^{2}$. Using integration by parts and the fact that $G$ vanishes on the boundary $x_{2}=0$ we obtain the estimate

$$
\|w-u\|_{L^{2}\left(U_{0}^{\prime}\right)} \leqslant C \varepsilon^{1 / 2}\|f\|_{L^{2}(\Omega)}+C r\|w-u\|_{L^{2}\left(U_{0}\right)}
$$

where $C$ is independent of $\varepsilon$ and $r$. The neighborhood $U_{0}^{\prime}$ of $x_{0}$ is the inverse image of $B_{r / 2}$. The boundary $\partial \Omega$ can be covered by a finite number of neighborhoods $\left\{U_{j}^{\prime}\right\}$ so that the number of intersecting neighborhoods $\left\{U_{j}\right\}$ at each point is less than 4 (cf. [15]). Setting $U=\cup_{j} U_{j}^{\prime}$ and using a partition of unity we obtain

$$
\|w-u\|_{L^{2}(U)} \leqslant C_{1} r\|w-u\|_{L^{2}(U)}+C_{2}\|w-u\|_{L^{2}(\Omega-U)}
$$

where $C_{1}$ is independent of $r$; hence (2.1) follows.

The main result of this paper is

THEOREM 2.1. Let $\Omega$ be a bounded domain in $R^{2}$ with smooth boundary $\partial \Omega$ and let $w$ and $u$ be the solutions of (1.2), (1.2') and (1.3), (1.3') respectively. Then there exists a constant $C$ such that for all $\varepsilon>0$ and $f \in L^{2}(\Omega)$, 


$$
\|w-u\|_{L^{2}(\Omega)} \leqslant C \varepsilon^{1 / 2}\|f\|_{L^{2}(\Omega)}
$$

Proof. Using the interior estimates of A. Friedman [6], we have for any subdomain $\Omega_{0}$ of $\Omega$, such that $\bar{\Omega}_{0} \subset \Omega$, the estimate

$$
\|w-u\|_{L^{2}\left(\Omega_{0}\right)} \leqslant C \varepsilon^{1 / 2}\|f\|_{L^{2}(\Omega)},
$$

where $C$ is a constant independent of $\varepsilon$ and $f$, but $C$ depends on $\operatorname{dist}\left(\Omega_{0}, \partial \Omega\right)$. Combining (2.1) and (2.7), inequality (2.6) follows.

The following results can be proved by the same methods and the results of A. Friedman [6].

THEOREM 2.2. Let $w$ and $u$ be the solutions of (1.2) and (1.3) with boundary conditions

$$
w=\partial^{2} w / \partial n^{2}=0 \quad \text { on } \partial \Omega,
$$

or

$$
w=\Delta w=0 \quad \text { on } \partial \Omega,
$$

or

$$
w=\partial \Delta w / \partial n=0 \quad \text { on } \partial \Omega
$$

and

$$
u=0 \quad \text { on } \partial \Omega \text {. }
$$

Then (2.6) holds.

TheOREM 2.3. Let $Q_{T}$ be the cylinder $Q_{T}=\Omega \times(0, T], B_{T}=\partial \Omega \times(0, T]$. Let $w$ be the solution of $\partial w / \partial t+L w=f(x, t)$ in $Q_{T}$ satisfying $\left(1.2^{\prime}\right),(2.8)$, (2.9) or (2.10) and (2.11) on $B_{T}, w(x, 0)=0$ in $\Omega$, and let $u$ be the solution of $\partial u / \partial t+M u=f(\mathbf{x}, t)$ in $Q_{T}$ satisfying (2.11) on $B_{T}$ and $u(\mathbf{x}, 0)=0$ in $\Omega$. Then

$$
\left(\int_{0}^{T} \int_{\Omega}|w(\mathbf{x}, t)-u(\mathbf{x}, t)|^{2} d \mathbf{x} d t\right)^{1 / 2} \leqslant C \varepsilon^{1 / 2}\left(\int_{0}^{T} \int_{\Omega}|f(\mathbf{x}, t)|^{2} d \mathbf{x} d t\right)^{1 / 2} .
$$

This result improves the results of J. L. Lions [12, p. 287], where

$$
\|w-u\|_{L^{2}\left(Q_{T}\right)}=O\left(\varepsilon^{1 / 4}\right)
$$

and A. Friedman [6] by extending his estimates up to the boundary.

REMARK. The estimate (2.6) is sharp and can be verified in the case of a clamped, uniform circular plate. It is probably not sharp in case boundary conditions (2.8), (2.9) or (2.10) replace $\left(1.2^{\prime}\right)$. The method of the present paper can be used to obtain sharper results for the various boundary value problems.

\section{REFERENCES}

1. M. S. Baouendi and C. Goulaouic, Régularité et théorie spectrale pour une classe d' opérateurs elliptiques dégéérés, Arch. Rational Mech. Anal. 34 (1969), 361-379. MR 40 \#3085; erratum, 41, p. 1964.

2. C. Bardos, D. Brézis and H. Brézis, Perturbationes singulieres et prolongements maximaux d'operateurs positifs, Arch. Rational Mech. Anal. 53 (1973/74), 69-100. MR 50 \# 745.

3. P. Bolley and J. Camus, Sur une classe d'opérateurs elliptiques et dégénérés a plusieurs variables, Bull. Soc. Math. France Mém. 34 (1973), 55-140. 
4. N. Dunford and J. T. Schwartz, Linear operators. I. General theory, Pure and Appl. Math., vol. 7, Interscience, New York, 1958. MR 22 \#8302.

5. A. Friedman, Partial differential equations, Holt, Rinehart and Winston, New York, 1969.

6. - Singular perturbations for partial differential equations, Arch. Rational Mech. Anal. 29 (1968), 289-303. MR 37 \# 1754.

7. W. M. Greenlee, Rate of convergence in singular perturbations, Ann. Inst. Fourier (Grenoble) 18 (1968), fasc. 2, 135-191, (1969). MR 39 \#3133.

8. E. Hille, Analytic function theory. Vol. II, Introduction to Higher Math., Blaisdell, Waltham, Mass., 1962. MR 34 \# 1490.

9. D. Huet, Singular perturbations of elliptic problems, Ann. Mat. Pura Appl. (4) 95 (1973), 77-114. MR 48 \#6631.

10. F. John, The transition from thin plate to membrane in the case of a plate under uniform tension, Continuum Mechanics and Related Problems of Analysis, Moscow, 1972.

11. L. Landau and E. Lifschitz, Continuum mechanics, OGIZ, Moscow, 1948; English transl., Pergamon Press, London; Addison-Wesley, Reading, Mass., 1958. MR 10, 582; 19, 1230.

12. J.-L. Lions, Perturbations singulières dans les problémes aux limites et en contrôles optimal, Lecture Notes in Math., Springer-Verlag, Berlin, 1973.

13. M. I. Visik and V. V. Grusin, Boundary value problems for elliptic equations degenerate on the boundary of a domain, Mat. Sb. 80 (122) (1969), 455-491 = Math. USSR Sb. 9 (1969), 423-454. MR 41 \#2212.

14. M. I. Visik and L. A. Ljusternik, Regular degeneration and boundary layer for linear differential equations with a small parameter, Uspehi Mat. Nauk 12 (1957), no. 5 (77), 3-122; English transl., Amer. Math. Soc. Transl. (2) 20 (1962), 239-364. MR 20 \#2539; 25 \#322.

15. J. J. Kohn and L. Nirenberg, Degenerate elliptic-parabolic equations of second order, Comm. Pure Appl. Math. 20 (1967), 797-872. MR 38 \#2437.

\section{Department of Mathematics, University of Kentucky, LeXington, Kentucky 40506}

Current address: Department of Mathematics, Rensselaer Polytechnic Institute, Troy, New York 12181 